Article

\title{
Towards the Provision of Accurate Atomic Data for Neutral Iron
}

\author{
Andrew Conroy ${ }^{1, * \mathbb{D}}$, Catherine Ramsbottom ${ }^{1}$, Connor Ballance ${ }^{1}$ and Francis Keenan ${ }^{2}$ \\ 1 Centre for Theoretical Atomic, Molecular and Optical Physics, Queen's University Belfast, University Road, \\ Belfast BT7 1NN, UK; c.ramsbottom@qub.ac.uk (C.R.); C.Ballance@qub.ac.uk (C.B.) \\ 2 Astrophysics Research Centre, Queen's University Belfast, University Road, Belfast BT7 1NN, UK; \\ F.Keenan@qub.ac.uk \\ * Correspondence: aconroy03@qub.ac.uk
}

Received: 30 June 2018; Accepted: 21 August 2018; Published: 25 August 2018

\begin{abstract}
The rich emission and absorption line spectra of Fe I may be used to extract crucial information on astrophysical plasmas, such as stellar metallicities. There is currently a lack, in quality and quantity, of accurate level-resolved effective electron-impact collision strengths and oscillator strengths for radiative transitions. Here, we discuss the challenges in obtaining an accurate model of the neutral iron atom and compare our theoretical fine-structure energy levels with observation for several increasingly large models. Radiative data is presented for several transitions for which the atomic data is accurately known.
\end{abstract}

Keywords: atomic data; iron; oscillator strengths

\section{Introduction}

The extraction of information about plasmas from their spectra is made possible by accurate collisional-radiative modelling. This relies on the availability of accurate atomic data such as radiative transition rates and electron-impact excitation cross-sections. When considering astrophysical plasmas, we pay particular attention to the iron-peak species, whose abundance is owed to their nuclear stability. Moreover, open d-shell species present in such plasmas will produce rich spectra due to the number of ways in which the electronic angular momenta can couple to form different fine-structure levels. At the intersection of the iron-peak and open d-shell species lies neutral iron. In this work, we seek a description of the structure of the neutral iron atom and accurate values for the oscillator strengths for radiative transitions, defined (for dipole transitions) by Hilborn [1], with the aim of calculating accurate Maxwell-averaged electron-impact collision strengths (effective collision strengths) among the fine-structure levels of Fe I.

Despite its rich spectrum, complete and comprehensive atomic data for Fe I are lacking. For example, NIST provides accurate (to within 3\%) oscillator strengths for only 149 fine-structure transitions [2]. These include the $524.71 \mathrm{~nm}$ and $525.02 \mathrm{~nm}$ lines, whose ratio has been used in magnetic field and temperature diagnostics [3]. In terms of collisional data, the first and only set of R-matrix electron-impact excitation collision strengths was published in 2017 [4], albeit term-resolved rather than level-resolved. This lack of radiative and collisional data for fine-structure transitions prevents us from fully exploiting the Fe I spectrum. The absence of theoretical results is due to the difficulty of obtaining a sufficiently good structure of the atom. For neutral systems, the non-central correlation interaction between electrons is comparable to the central Coulomb interaction between electrons and the nucleus and a large configuration interaction $(\mathrm{CI})$ basis is required to accurately describe the structure. Conversely, the computational expense of a Dirac R-matrix calculation to obtain collisional data limits the size of CI basis we may use. The challenge we face is to describe the structure of the atom using a sufficiently small CI basis. 
As a consequence of the presence of numerous absorption lines in the spectra of late-type stars, neutral iron provides chemical abundance diagnostics, with the ratio of the Fe I content to the hydrogen content, $[\mathrm{Fe} / \mathrm{H}]$, sometimes being used to describe the metallicity of a star [5]. The extraction of information from spectra on the abundance of neutral iron relies on the accurate population modelling of as many of its fine-structure levels as possible and, in particular, the availability of highly reliable oscillator strengths for many transitions.

In this paper, we discuss the difficulties in obtaining a sufficiently good structure for the iron atom. We present theoretical energy levels in Section 2 and oscillator strengths in Section 3, obtained from different structure calculations for neutral iron. These are compared to experimental data, paying particular attention to the final model presented which is currently being used to generate a set of level-resolved collisional data for this species.

\section{Atomic Structure}

To obtain oscillator strengths for radiative transitions and collision strengths for electron-impact (de-)excitations, we required a suitable description of the neutral iron atom. This was obtained using P. Norrington's updated ${ }^{1}$ version of the fully relativistic General-Purpose Relativistic Atomic Structure Program $\left(\mathrm{GRASP}^{0}\right.$ ) [6,7]. GRASP ${ }^{0}$ has recently been used to model atomic systems as complex as neutral molybdenum [8] and neutral tungsten [9] and the quality of the collisional and radiative data obtained verified by comparison of synthetic spectra and experiment. For this reason, GRASP ${ }^{0}$ was a reasonable choice of structure package for this calculation.

GRASP $^{0}$ finds the approximate eigenstates of the Dirac-Coulomb Hamiltonian, $\hat{H}$, given by (in atomic units)

$$
\hat{H}=\sum_{m=1}^{N}\left(-i c \boldsymbol{\alpha} \cdot \nabla_{m}+\left(\beta-I_{4}\right) c^{2}-\frac{Z}{r_{m}}\right)+\sum_{m>n} \frac{1}{\left|\mathbf{r}_{m}-\mathbf{r}_{n}\right|},
$$

where $N=Z=26$ for Fe I. Eigenstates of this Hamiltonian may be grouped into $J \pi$-symmetry blocks, where $J$ is the total angular momentum of the state and $\pi$ its parity. This problem is solved using an extensive basis set of configuration state functions (CSFs) obtained using the multi-configurational Dirac-Fock method (MCDF). Extended average level (EAL) calculations were performed on different configuration sets, where a set of variational one-electron orbitals are found such that the weighted trace (with each $J \pi$ level assigned weight $2 J+1$, the degeneracy of the level) of the Dirac-Coulomb Hamiltonian is minimised.

Obtaining a good structure for this neutral, open d-shell system is difficult. In the MCDF equations used to obtain the orbitals, correlation interactions are spherically averaged. The non-central component of this interaction is accounted for by using an extensive basis of CSFs with which to diagonalise the Hamiltonian matrix. For a neutral system, the correlation interaction is comparable to the central Coulomb interaction between electrons and the nucleus, and a small (in terms of the CSF basis size used) model of the atom cannot be expected to yield an accurate structure. However, the computational expense of any Dirac R-matrix calculation to obtain scattering data prohibits the use of very large models. To this end, the configuration set employed in a model must be carefully chosen so that correlation effects are reasonably well-described, while avoiding excessively large CSF bases. As we will see, if we limit the number of basis functions used, some states are more accurately described than others.

Another major problem we may encounter is the failure of the MCDF equations to converge for an orbital. In some cases, we may achieve convergence by using a different trial function for this orbital. In our work, one of the obstacles encountered was extending the orbital set beyond $4 p$ by introducing a $4 \mathrm{~d}$ orbital, which often resulted in convergence problems for both the $4 \mathrm{~d}$ and $3 \mathrm{~d}$ orbitals.

1 This updated version of GRASP $^{0}$ is freely available at http:// connorb.freeshell.org/. 
Presently, our working model of the Fe I atom consists of 21 non-relativistic configurations, giving rise to 5955 relativistic configurations and fine-structure levels, which we call Model 6. However, Models 1 to 5 are also discussed here, to illustrate the difficulties in obtaining a sufficiently good structure. Table 1 shows the configurations included in each model. Note that all configurations listed have a closed core, all orbitals are spectroscopic and each model includes the configurations from the previous model as well as those listed in the relevant column of the table.

In Model 1 (6 configurations and 995 levels), we included the ground configuration $\left(3 \mathrm{p}^{6} 3 \mathrm{~d}^{6} 4 \mathrm{~s}^{2}\right)$, expanding this into a 6-configuration model by allowing promotions from the 4 s orbital to the $3 \mathrm{~d}$ and 4 p orbitals. For Model 2 (eight configurations and 1162 levels), we allowed double excitations from the $3 p$ orbital to the $3 \mathrm{~d}$ orbital for two even configurations to generate more even parity states, with the hope of lowering the energy eigenvalues of the even levels. It was thought unnecessary to allow double excitations in odd configurations at this stage, as their energies relative to the ground were already too low. In Model 3 (12 configurations and 4644 levels ), we expanded the orbital set to include a $4 \mathrm{~d}$ orbital. The $3 \mathrm{~d}^{8} 4 \mathrm{~d}^{6}$ configuration was included to allow for the convergence of the $3 \mathrm{~d}$ and $4 \mathrm{~d}$ orbitals in the MCDF calculation. The importance of including a $4 \mathrm{~d}$ orbital lies in the fact that the previous configurations included only one d-subshell. In the MCDF-EAL method, we use a unique orbital basis and rely on CI to account for correlation effects instead of using different orbitals for different configurations. A 3d orbital that describes some states well may describe others poorly. By supplementing the CSF basis with $4 \mathrm{~d}$-occupied configurations, we hope to obtain a more accurate description of the $3 \mathrm{~d}$ electrons in each configuration and hence a better estimate of the eigenfunctions of the Dirac-Coulomb Hamiltonian.. Models 4 (15 configurations and 4842 levels) and 5 (17 configurations and 5396 levels) introduced a 5s and 5p orbital, respectively. In Model 6 (21 configurations), we allowed excitations from the $3 p$ orbital to the $3 \mathrm{~d}$ orbital for four configurations. At this stage, configurations were added one by one in order to identify important CSFs and to reduce the size of the final model. The variability of the MCDF orbitals with the addition of new configurations necessitated such a careful approach. Configurations were only retained if they had a significant effect on the accuracy of the model.

Table 1. Six models of the iron atom, described by their configuration sets. Each model adds to the previous configuration set.

\begin{tabular}{cccccc}
\hline Model 1 & Model 2 & Model 3 & Model 4 & Model 5 & Model 6 \\
\hline $3 p^{6} 3 d^{6} 4 s^{2}$ & $3 p^{4} 3 d^{8} 4 s^{2}$ & $3 d^{8} 4 d^{6}$ & $3 p^{6} 3 d^{6} 4 s 5 s$ & $3 p^{6} 3 d^{7} 5 p$ & $3 p^{4} 3 d^{9} 4 p$ \\
$3 p^{6} 3 d^{6} 4 s 4 p$ & $3 p^{4} 3 d^{9} 4 s$ & $3 p^{6} 3 d^{7} 4 d$ & $3 p^{6} 3 d^{7} 5 s$ & $3 p^{6} 3 d^{6} 5 p^{2}$ & $3 p^{4} 3 d^{9} 4 d$ \\
$3 p^{6} 3 d^{7} 4 s$ & & $3 p^{6} 3 d^{6} 4 s 4 d$ & $3 p^{6} 3 d^{6} 5 s^{2}$ & & $3 p^{4} 3 d^{9} 5 s$ \\
$3 p^{6} 3 d^{7} 4 p$ & & $3 p^{6} 3 d^{6} 4 p 4 d$ & & & $3 p^{4} 3 d^{8} 5 s^{2}$ \\
$3 p^{6} 3 d^{8}$ & & & & & \\
$3 p^{6} 3 d^{6} 4 p^{2}$ & & & & & \\
\hline
\end{tabular}

Table 2 shows the NIST values of selected fine-structure energy levels [2], relative to the ground state, alongside the percentage differences with those obtained in these calculations. This includes the first 30 fine structure levels and the first ${ }^{7} \mathrm{P}^{\mathrm{o}}$ and ${ }^{5} \mathrm{P}^{\mathrm{o}}$ terms. By careful choice of configuration state basis, we have achieved an average percentage discrepancy from NIST of $7.2 \%$ over 275 fine-structure levels. The addition of extra even configurations in Model 2 was effective in pushing the ground state downwards in energy, which can be seen in the improvement of the energies of the odd levels, relative to the ground. In Model 3, we can see a dramatic change in the accuracies of levels dominated by $3 d^{7} 4$ s configuration states with the introduction of a $4 \mathrm{~d}$ orbital. Clearly, the $3 \mathrm{~d}$ orbitals vary significantly between $3 d^{6} 4 s^{2}, 3 d^{6} 4 s 4 p$ and $3 d^{7} 4 s$ configuration states and a compromise orbital cannot simultaneously give a good description of all of these states; CI with 4 d-occupied states was essential in obtaining an adequate description of these levels. The addition of $5 \mathrm{~s}$ and $5 \mathrm{p}$ orbitals in Models 4 and 5 augmented the CI basis used and allowed for a general improvement in all levels 
shown, excluding the ${ }^{3} \mathrm{P}$ term. The eigenvectors corresponding to the $3 \mathrm{~d}^{6} 4 \mathrm{~s}^{2}{ }^{3} \mathrm{P}$ levels in these models were heavily mixed with $3 \mathrm{~d}^{7} 4 \mathrm{~s}$ configuration states and matching our calculated ${ }^{3} \mathrm{P}$ terms with NIST was not straightforward. This issue was resolved by Model 6, where a selection of doubly excited configurations were introduced. Particular attention was paid to the ${ }^{5} \mathrm{~F}$ term when choosing which configurations to retain in our model. The addition of these configurations resulted in improvements across all energies shown in Table 2 with respect to their separation from the ground level. In Model 6, over the levels shown in Table 2, discrepancies differ from term to term, varying from around $-3 \%$ for the ${ }^{7} \mathrm{D}^{\mathrm{o}}$ term to around $+25 \%$ for ${ }^{3} \mathrm{H}$. Although we expect the energies to converge to the observed values as the CSF basis size increases, we cannot afford to describe all levels equally accurately. This is shown in the decreased accuracy of the levels of the ground term, ${ }^{5} \mathrm{D}$, in Model 6 compared to Model 1.

Table 2. Percentage differences of the energy levels from different GRASP ${ }^{0}$ (General-Purpose Relativistic Atomic Structure Program) structures with the experimental NIST (National Institute of Standards and Technology) values.

\begin{tabular}{|c|c|c|c|c|c|c|c|c|}
\hline \multirow{2}{*}{ No. } & \multirow{2}{*}{ Level } & \multirow{2}{*}{ NIST } & \multicolumn{6}{|c|}{ GRASP $^{0}$} \\
\hline & & & Model 1 & Model 2 & Model 3 & Model 4 & Model 5 & Model 6 \\
\hline 1 & $3 d^{6} 4 s^{2}{ }^{5} D_{4}$ & 0.00000 & - & - & - & - & - & - \\
\hline 2 & $3 d^{6} 4 s^{2}{ }^{5} D_{3}$ & 0.00379 & 0.8 & 1.0 & -9.9 & -9.6 & -9.0 & -7.2 \\
\hline 3 & $3 d^{6} 4 s^{2}{ }^{5} D_{2}$ & 0.00642 & 1.7 & 1.4 & -9.1 & -8.8 & -8.3 & -6.6 \\
\hline 4 & $3 d^{6} 4 s^{2}{ }^{5} D_{1}$ & 0.00809 & 2.4 & 1.7 & -8.6 & -8.3 & -7.7 & -6.1 \\
\hline 5 & $3 d^{6} 4 s^{2}{ }^{5} D_{0}$ & 0.00891 & 2.6 & 1.9 & -8.4 & -8.1 & -7.5 & -5.9 \\
\hline 6 & $3 d^{7} 4 s^{5} F_{5}$ & 0.06314 & 489.2 & 522.4 & -77.5 & -67.5 & -53.2 & -10.8 \\
\hline 7 & $3 d^{7} 4 s^{5} F_{4}$ & 0.06722 & 457.9 & 489.0 & -72.9 & -63.7 & -50.1 & -10.1 \\
\hline 8 & $3 d^{7} 4 s^{5} F_{3}$ & 0.07042 & 435.4 & 465.5 & -69.7 & -60.9 & -47.9 & -9.7 \\
\hline 9 & $3 d^{7} 4 s^{5} F_{2}$ & 0.07277 & 420.8 & 449.6 & -67.6 & -58.9 & -46.4 & -9.4 \\
\hline 10 & $3 d^{7} 4 s^{5} F_{1}$ & 0.07431 & 411.4 & 439.8 & -66.2 & -57.7 & -45.5 & -9.2 \\
\hline 11 & $3 d^{7} 4 s^{3} F_{4}$ & 0.10914 & 294.9 & 313.5 & -27.9 & -27.0 & -17.8 & 6.3 \\
\hline 12 & $3 d^{7} 4 s^{3} F_{3}$ & 0.11446 & 279.8 & 297.7 & -28.1 & -25.9 & -17.3 & 5.9 \\
\hline 13 & $3 d^{7} 4 s^{3} F_{2}$ & 0.11818 & 270.6 & 287.6 & -30.3 & -25.2 & -16.7 & 5.7 \\
\hline 14 & $3 d^{7} 4 s^{5} P_{3}$ & 0.15993 & 225.6 & 231.0 & -31.6 & -27.7 & -21.8 & -5.6 \\
\hline 15 & $3 d^{7} 4 s^{5} P_{2}$ & 0.16154 & 223.1 & 228.5 & -31.2 & -27.3 & -21.4 & -5.4 \\
\hline 16 & $3 d^{7} 4 s^{5} P_{1}$ & 0.16337 & 220.2 & 225.5 & -30.9 & -27.0 & -21.3 & -5.4 \\
\hline 17 & $3 d^{6} 4 s^{2}{ }^{3} P_{2}$ & 0.16747 & 29.7 & 11.8 & 29.6 & 31.5 & 30.8 & 9.0 \\
\hline 18 & $3 d^{6} 4 s 4 p^{7} D_{5}^{O}$ & 0.17634 & -23.6 & -8.0 & -6.4 & -6.5 & -4.2 & -3.3 \\
\hline 19 & $3 d^{6} 4 s^{2}{ }^{3} H_{6}$ & 0.17670 & 10.9 & 18.3 & 63.3 & 30.0 & 24.9 & 24.3 \\
\hline 20 & $3 d^{6} 4 s^{2}{ }^{3} P_{1}$ & 0.17818 & 26.6 & 11.8 & 26.3 & 27.3 & 29.3 & 7.4 \\
\hline 21 & $3 d^{6} 4 s 4 p^{7} D_{4}^{o}$ & 0.17827 & -23.1 & -7.9 & -6.5 & -6.5 & -4.3 & -3.4 \\
\hline 22 & $3 d^{6} 4 s^{2}{ }^{3} H_{5}^{4}$ & 0.17880 & 10.7 & 17.9 & 29.0 & 29.5 & 24.2 & 23.8 \\
\hline 23 & $3 d^{6} 4 s 4 p^{7} D_{3}^{o}$ & 0.18004 & -22.9 & -7.8 & -6.5 & -6.5 & -4.3 & -3.4 \\
\hline 24 & $3 d^{6} 4 s^{2}{ }^{3} H_{4}$ & 0.18031 & 10.5 & 17.1 & 28.8 & 28.4 & 24.4 & 23.1 \\
\hline 25 & $3 d^{6} 4 s 4 p^{7} D_{2}^{o}$ & 0.18146 & -22.6 & -7.7 & -6.5 & -6.5 & -4.3 & -3.4 \\
\hline 26 & $3 d^{6} 4 s 4 p^{7} D_{1}^{o}$ & 0.18243 & -22.5 & -7.6 & -6.5 & -7.5 & -4.3 & -3.4 \\
\hline 27 & $3 d^{6} 4 s^{2}{ }^{3} P_{0}$ & 0.18260 & 26.3 & 11.8 & 25.6 & 26.3 & 27.8 & 6.9 \\
\hline 28 & $3 d^{6} 4 s^{2}{ }^{3} F_{4}$ & 0.18810 & 19.2 & 13.5 & 21.8 & 21.6 & 20.6 & 19.0 \\
\hline 29 & $3 d^{6} 4 s^{2}{ }^{3} F_{3}$ & 0.19022 & 19.0 & 12.9 & 21.5 & 21.3 & 20.3 & 18.3 \\
\hline 30 & $3 d^{6} 4 s^{2}{ }^{3} F_{2}$ & 0.19172 & 18.9 & 12.8 & 21.2 & 21.0 & 20.0 & 18.3 \\
\hline 44 & $3 d^{6} 4 s 4 p^{7} P_{4}^{o}$ & 0.21607 & -20.4 & -7.9 & -6.8 & -6.7 & -6.3 & -5.3 \\
\hline 47 & $3 d^{6} 4 s 4 p^{7} P_{3}^{{ }^{\circ}}$ & 0.22035 & -19.8 & -7.6 & -6.7 & -6.6 & -6.2 & -5.2 \\
\hline 50 & $3 d^{6} 4 s 4 p^{7} P_{2}^{o}$ & 0.22332 & -19.4 & -7.3 & -6.6 & -6.5 & -6.1 & -5.1 \\
\hline 73 & $3 d^{6} 4 s 4 p^{5} P_{3}^{0}$ & 0.26478 & -10.8 & -0.6 & -7.4 & -6.8 & -6.5 & -4.9 \\
\hline 78 & $3 d^{6} 4 s 4 p^{5} P_{2}^{o}$ & 0.26854 & -10.5 & -0.5 & -7.4 & -6.8 & -6.5 & -4.9 \\
\hline 79 & $3 d^{6} 4 s 4 p^{5} P_{1}^{o}$ & 0.27094 & -10.3 & -0.4 & -7.4 & -6.9 & -6.5 & -4.9 \\
\hline
\end{tabular}




\section{Radiative Data}

Oscillator strengths for fine-structure transitions were calculated using the OSCL package in GRASP $^{0}$. In Table 3, we present electric dipole (E1) oscillator strengths for Models 1 to 6, in length form, for a selection of transitions among the fine-structure levels shown in Table 2 with accurately known radiative data [2]. In Model 6, we see that the addition of new configurations makes little difference to the oscillator strengths obtained for transitions among the first 30 levels. Discrepancies with NIST for the oscillator strengths from Model 6 vary, with the $23-2$ transition $6.5 \%$ above the NIST value, whereas the $25-2$ transition is almost six times greater. Transitions from the ${ }^{7} \mathrm{Po}^{\mathrm{o}}$ term to the ${ }^{5} \mathrm{D}$ term suffered with the addition of configurations in Model 6, with the average discrepancy from NIST falling from $10.9 \%$ in Model 5 to $25.4 \%$ in Model 6. For atomic systems as complex as neutral iron, we expect that billions of CSFs are required to obtain fully converged oscillator strengths [10]. For this reason, it is unsurprising that some of the oscillator strengths listed may change dramatically with the expansion of the CSF basis, given that Model 6 contains only thousands of CSFs. For six of the 11 transitions listed in Table 3, we have achieved a reasonable agreement with NIST of within $20 \%$.

Table 3. E1 oscillator strengths from various models compared with the accurate NIST values, where $a-b \equiv a \times 10^{-b}$.

\begin{tabular}{ccccccccc}
\hline \multirow{2}{*}{ Wavelength /nm } & \multirow{2}{*}{ Transition $(j-i)$} & \multicolumn{7}{c}{ Oscillator Strength } \\
\cline { 3 - 8 } & & NIST & Model 1 & Model 2 & Model 3 & Model 4 & Model 5 & Model 6 \\
\hline 525.50 & $25-4$ & $5.74-6$ & $6.80-7$ & $8.62-7$ & $4.14-6$ & $5.03-6$ & $6.56-6$ & $6.38-6$ \\
525.02 & $26-5$ & $1.15-5$ & $1.21-6$ & $1.62-6$ & $6.49-6$ & $7.09-6$ & $1.02-5$ & $1.03-5$ \\
524.71 & $23-3$ & $2.26-6$ & $2.71-7$ & $6.43-8$ & $2.42-6$ & $3.19-6$ & $3.68-6$ & $3.37-6$ \\
522.55 & $26-4$ & $5.42-6$ & $5.17-7$ & $7.14-7$ & $2.50-6$ & $2.45-6$ & $3.88-6$ & $4.02-6$ \\
520.46 & $25-3$ & $9.31-6$ & $1.13-6$ & $1.49-6$ & $5.06-6$ & $5.63-6$ & $8.37-6$ & $8.55-6$ \\
516.89 & $23-2$ & $1.53-5$ & $2.15-6$ & $2.76-6$ & $9.45-6$ & $1.13-5$ & $1.62-5$ & $1.63-5$ \\
512.77 & $25-2$ & $1.07-7$ & $9.21-8$ & $9.27-8$ & $3.05-7$ & $7.01-7$ & $7.53-7$ & $7.04-7$ \\
511.04 & $21-1$ & $1.93-5$ & $2.90-6$ & $3.64-6$ & $1.30-5$ & $1.61-5$ & $2.24-5$ & $2.23-5$ \\
425.83 & $47-3$ & $9.66-6$ & $4.10-7$ & $5.30-7$ & $1.87-5$ & $1.42-5$ & $1.03-5$ & $7.30-6$ \\
423.27 & $50-4$ & $3.93-6$ & $1.60-7$ & $2.10-7$ & $7.17-6$ & $5.13-6$ & $3.59-6$ & $2.61-6$ \\
421.62 & $44-1$ & $4.90-5$ & $2.28-6$ & $2.88-6$ & $8.90-5$ & $7.33-5$ & $5.75-5$ & $4.16-5$ \\
\hline
\end{tabular}

\section{Conclusions and Outlook}

In this paper, we have contrasted the amount of information contained in neutral iron spectra with the lack of appropriate, high quality atomic data for this species, and discussed the challenges in obtaining a sufficiently good structure for the neutral iron atom with which to obtain theoretical results, keeping in mind that future Dirac R-matrix calculations prohibit excessively large structures. We have compared the atomic data obtained from structure calculations on six increasingly large models.

Our present working model is currently being used to calculate electron-impact collision strengths for Fe I, using the Dirac R-matrix method, with 300 target levels. All but 25 of these levels could be matched to NIST values by their dominant eigenvector component and LSJ $\pi$ term. The energies of the 275 NIST-matched levels vary, on average, from NIST values by an absolute percentage of 7.2\%. On average, the energies obtained were 3.6\% lower than the NIST values. In the R-matrix calculation, energy levels have been shifted to the NIST values, with the 25 unmatched levels shifted down by $3.6 \%$. The accuracy of the levels included will be seen once another R-matrix calculation has been carried out with unshifted energies, where we hope to see that the only difference is in the positions of the resonances in the collision strengths. In the future, additional structures will also be used to generate level-resolved collisional data. This is important due to the lack of level-resolved collision strengths for this species. Several sets of collisional data will allow a meaningful error analysis of the atomic data calculated in this investigation. 
Author Contributions: Conceptualization, C.A.R., C.P.B. and F.P.K.; Investigation, A.T.C.; Supervision, C.A.R., C.P.B. and F.P.K.; Writing-Original Draft, A.T.C.; Writing-Review \& Editing, C.A.R., C.P.B. and F.P.K.

Funding: This work is supported by funding from the STFC ST/P000312/1 QUB Astronomy Observation and Theory Consolidated Grant.

Acknowledgments: All calculations were undertaken on a computer cluster at Queen's University Belfast.

Conflicts of Interest: The authors declare no conflict of interest.

\section{References}

1. Hilborn, R.C. Einstein coefficients, cross sections, f values, dipole moments, and all that. arXiv 2002, arXiv:physics/0202029.

2. Kramida, A.; Ralchenko, Y.; Reader, J.; NIST ASD Team. NIST Atomic Spectra Database (Version 5.5.6). 2018. Available online: https:/ / physics.nist.gov/asd (accessed on 19 June 2018).

3. Holzreuter, R.; Solanki, S.K. Three-dimensional non-LTE radiative transfer effects in Fe lines - I. Flux sheet and flux tube geometries. Astron. Astrophys. 2012, 547, A46. [CrossRef]

4. Bautista, Manuel A.; Lind, K.; Bergemann, M. Photoionization and electron impact excitation cross sections for Fe. Astron. Astrophys. 2017, 606, A127. [CrossRef]

5. Bergemann, M.; Lind, K.; Collet, R.; Magic, Z.; Asplund, M. Non-LTE line formation of Fe in late-type stars I. Standard stars with 1D and 3D model atmospheres. Mon. Not. R. Astron. Soc. 2012, 427, 27-49. [CrossRef]

6. Dyall, K.P.; Grant, I.P.; Johnson, C.T.; Parpia, F.A.; Plummer, E.P. GRASP: A general-purpose relativistic atomic stucture program. Comput. Phys. Commun. 1996, 94, 249. [CrossRef]

7. Parpia, F.A.; Grant, I.P. Software for relativistic atomic theory: The GRASP project at Oxford. J. Phys. IV Fr. 1991, 1, C1:33-C1:46. [CrossRef]

8. Smyth, R.T.; Johnson, C.A.; Ennis, D.A.; Loch, S.D.; Ramsbottom, C.A.; Ballance, C.P. Relativistic R-matrix calculations for the electron-impact excitation of neutral molybdenum. Phys. Rev. A 2017, 96, 042713. [CrossRef]

9. Smyth, R.T.; Ballance, C.P.; Ramsbottom, C.A.; Johnson, C.A.; Ennis, D.A.; Loch, S.D. Dirac R-matrix calculations for the electron-impact excitation of neutral tungsten providing noninvasive diagnostics for magnetic confinement fusion. Phys. Rev. A 2018, 97, 052705. [CrossRef]

10. Aggarwal, K.M. Discrepancies in Atomic Data and Suggestions for Their Resolutions. Atoms 2017, 5, 37. [CrossRef]

(C) 2018 by the authors. Licensee MDPI, Basel, Switzerland. This article is an open access article distributed under the terms and conditions of the Creative Commons Attribution (CC BY) license (http:// creativecommons.org/licenses/by/4.0/). 